Materials Sciences Division, Lawrence Berkeley National Laboratory, and

Department of Materials Science and Engineering

University of California at Berkeley

\title{
EFFECT OF ORIENTATION ON THE IN VITRO FRACTURE TOUGHNESS OF DENTIN: THE ROLE OF TOUGHENING MECHANISMS
}

\author{
R. K. Nalla ${ }^{1}$ J. H. Kinney ${ }^{2}$ and R. O. Ritchie ${ }^{1,3}$ \\ ${ }^{1}$ Materials Sciences Division, Lawrence Berkeley National Laboratory, and \\ Department of Materials Science and Engineering, \\ University of California, Berkeley, CA 94720 \\ 2 Department of Preventive and Restorative Dental Sciences, \\ University of California, San Francisco, CA 94143 \\ ${ }^{3}$ Corresponding author: Tel: (510) 486-5798; Fax: (510) 486-4881 \\ E-mail address: RORitchie@lbl.gov (R. O. Ritchie)
}

November 2002

submitted to: Biomaterials

This work was supported in part by the National Institutes of Health, National Institute for Dental and Craniofacial Research under Grant No. P01DE09859. 


\title{
EFFECT OF ORIENTATION ON THE IN VITRO FRACTURE TOUGHNESS OF DENTIN: THE ROLE OF TOUGHENING MECHANISMS
}

\author{
R. K. Nalla ${ }^{1}$, J. H. Kinney ${ }^{2}$ and R. O. Ritchie ${ }^{1, *}$ \\ ${ }^{1}$ Materials Sciences Division, Lawrence Berkeley National Laboratory, and \\ Department of Materials Science and Engineering, \\ University of California, Berkeley, CA 94720 \\ ${ }^{2}$ Department of Preventive and Restorative Dental Sciences, \\ University of California, San Francisco, CA 94143
}

\begin{abstract}
Toughening mechanisms based on the presence of collagen fibrils have long been proposed for mineralized biological tissues like bone and dentin; however, no direct evidence for their precise role has ever been provided. Furthermore, although the anisotropy of mechanical properties of dentin with respect to orientation has been suggested in the literature, accurate measurements to support the effect of orientation on the fracture toughness of dentin are not available. To address these issues, the in vitro fracture toughness of dentin, extracted from elephant tusk, has been characterized using fatigue-precracked compact-tension specimens tested in Hank's Balanced Salt Solution at ambient temperature, with fracture paths perpendicular and parallel to the tubule orientations (and orientations in between) specifically being evaluated. It was found that the fracture toughness was lower where cracking occurred in the plane of the collagen fibers, as compared to crack paths perpendicular to the fibers. The origins of this effect on the toughness of dentin are discussed primarily in terms of the salient toughening mechanisms active in this material; specifically, the role of crack bridging, both from uncracked ligaments and by individual collagen fibrils, is considered. Estimates for the contributions from each of these mechanisms are provided from theoretical models available in the literature.
\end{abstract}

Keywords: Collagen, fracture toughness, tubules, orientation, toughening mechanisms, dentin.

\footnotetext{
* Corresponding author: Tel: (510) 486-5798; Fax: (510) 486-4881

E-mail address: RORitchie@lbl.gov (R. O. Ritchie)
} 


\section{Introduction}

Dentin is the most abundant mineralized tissue in the tooth. Similar in composition to bone, it is composed largely of type-I collagen fibrils and nanocrystalline apatite mineral [1]. The most striking microstructural feature is the dentinal tubule, cylindrical channels that course continuously from the dentin-enamel and cementum-enamel junctions to the pulp. A thin, highly mineralized cuff of peritubular dentin surrounds each tubule. The mineralized collagen fibrils are arranged orthogonal to the tubules, forming a planar, feltlike structure called the intertubular dentin matrix [2]. This highly oriented microstructure is believed to confer anisotropy to the mechanical properties, although the magnitude and orientation of the anisotropy is not well established. Indeed, after some five decades of research on the mechanical properties of dentin [e.g., 3-15], there is still little consistency in some of the basic questions that dictate its structural behavior.

Because the peritubular dentin is highly mineralized, it had long been suspected that dentin had a higher elastic modulus in the direction of the tubules. However, recent micromechanics arguments [12] and sensitive acoustic measurements [3] now support the view that the elastic properties are determined by the mineralized collagen fibers, and that dentin is therefore stiffest in an orientation perpendicular to the axes of the tubules. These more recent results have largely substantiated for human dentin what had been observed in an earlier study on Narwhal tusk dentin by Currey et al., namely, that there is a strong dependence of the elastic properties on the orientation of the mineralized collagen fibrils [15].

The observations that the orientation of the collagen fibrils affects the symmetry of the elastic properties suggest that their orientation might also influence fracture behavior. This is important as resistance to fracture in teeth is an issue of great clinical relevance. Exposed root surfaces often exhibit non-carious notches in the dentin just below the enamel-cementum junction, which can act as sites for unstable fracture. While cusp fractures are common in posterior teeth, the anterior teeth are more susceptible to fracture in the gingiva, severing the crown of the tooth. Although such fractures have not been investigated extensively, it is generally believed that they are catastrophic events induced by occlusal stresses. In light of this, some measure of the fracture resistance of dentin is necessary. 
Fracture mechanics provides an appropriate methodology to quantify the fracture resistance of dentin. Under linear-elastic conditions, fracture instability is reached when the stress intensity ahead of a pre-existing crack exceeds the fracture toughness, $K_{\mathrm{c}}$, of the material, i.e.,

$$
K=Y \sigma_{\text {app }}(\pi a)^{1 / 2}=K_{\mathrm{c}},
$$

where $\sigma_{\text {app }}$ is the applied (service) stress, $a$ is the crack length, and $Y$ is a function of geometry, crack size and shape (and of order unity). Alternatively, the fracture toughness can be expressed in terms of a critical value of the strain energy release rate, $G_{\mathrm{c}}$, defined as the change in potential energy per unit increase in crack area, where:

$$
G_{\mathrm{c}}=K_{\mathrm{c}}^{2} / E^{\prime}
$$

and where $E^{\prime}=E$, the elastic modulus in plane stress and $E /\left(1-v^{2}\right)$ in plane strain $(v$ is Poisson's ratio) [16].

To date, only a few studies have provided a quantitative evaluation of the fracture toughness of dentin. The earliest was by Rasmussen et al. [8,9] who used a "work of fracture" (defined as the work per unit area to generate new crack surface) to quantify the fracture resistance. These authors reported an orientation effect on the toughness of dentin in that the work of fracture was found to be lower for cracking perpendicular to the dentinal tubular direction, i.e., in the plane of the mineralized collagen fibrils, compared to all other directions. Such a result is consistent with the notion that crack bridging by the collagen fibrils could enhance the toughness along directions parallel to the tubule axes. Indeed, ref. [8] does indicate that crack propagation perpendicular to the tubules is more energetically favorable, consistent with the absence of fiber bridging in that direction. However, excessive scatter in their results makes such definitive conclusions difficult; moreover, no direct evidence of such bridging was presented.

A subsequent study, by el Mowafy et al. [10], was the first to utilize a fracture mechanics approach, using notched (but not precracked) compact-tension specimens to measure the fracture toughness of human dentin to be $K_{\mathrm{c}}=3.08 \mathrm{MPa} \sqrt{\mathrm{m}}$ (std. dvn. 0.33 $\mathrm{MPa} \vee \mathrm{m}$ ) for a single orientation parallel to the long axis of the tubules. In a similar vein, Iwamoto et al. [13] reported fracture toughness values for human dentin $(1.13 \pm 0.36$ $\mathrm{MPa} \sqrt{\mathrm{m}}$ to $2.02 \pm 0.18 \mathrm{MPa} \sqrt{\mathrm{m}}$, depending on orientation), using the so-called notchless 
triangular prism specimen geometry. The latter technique permits the use of very small samples, which allowed these authors to also show an effect of orientation on toughness; however, the accuracy of their $K_{\mathrm{c}}$ data may be deemed to be somewhat questionable in light of the non-standard nature of their toughness tests. Most recently, Imbeni et al. [14] used fatigue-precracked three-point bend bar samples (nominally conforming to ASTM standards) in order to determine an accurate measure of the in vitro fracture toughness of human dentin ${ }^{1}$. Measurements, made for a crack path perpendicular to the tubules in order to determine a worst-case value, yielded a fracture toughness of $K_{\mathrm{c}}=1.79 \mathrm{MPa} \sqrt{\mathrm{m}}$ (std. dvn. 0.1). Due to specimen size requirements, other orientations could not be examined.

These limited results suggest that the fracture resistance of dentin is anisotropic, although an accurate quantification of the variation in $K_{\mathrm{c}}$ with orientation is still lacking and the precise mechanisms underlying the effect are as yet unproven. Part of the problem has been the relatively small size of the specimens that can be made with human dentin, which makes it difficult to accurately address the role of orientation on toughness. To alleviate this problem in the present work, we have chosen to study elephant dentin, which permits the use of specimens of appropriate size to determine valid measurements of the fracture toughness in all relevant tubule, and hence collagen, orientations. Specifically, we address several critical hypotheses in a systematic evaluation of effect of orientation on the fracture toughness and of the crack-microstructure interactions in elephant dentin:

- What is the role of collagen fibril orientation on the toughness?

- Does fibril bridging occur in a mineralized tissue of similar composition to bone?

- Is the anisotropy of the fracture toughness consistent with such crack bridging?

- Do the tubules affect the toughness, either by blunting or deflecting cracks?

- Are there other salient mechanisms of toughening in dentin?

It is believed that some resolution of these issues provides insight into the functional role of microstructure, and specifically the collagen fibrils, in influencing the fracture of mineralized tissue.

\footnotetext{
${ }^{1}$ These authors demonstrated a marked effect of notch acuity on the toughness values, thereby indicating that earlier measurements of the fracture toughness of dentin, which used notched rather than pre-cracked
} 


\section{Materials and Experimental Procedures}

\subsection{Materials}

Recently fractured shards of elephant tusk from an adult male elephant (Loxodonta Africana) were used in the present study. The bulk of the tusk material, which is commonly referred to as ivory, is composed of dentin. A typical scanning electron micrograph of the microstructure of the material used is given in Fig. 1a, showing an orientation perpendicular to the long axis of the dentinal tubules. Akin to human dentin, the characteristic feature of this microstructure is the presence of tubules that are the result of odontoblast cell movement during dentin formation. These tubules sit in a matrix formed by the mineralization of type I collagen fibers deposited in the early stages of formation [17]. At this juncture, it is important to point out that although the microstructure is very similar to that of human dentin, there are some essential differences: the tubules are more elliptical in shape [17] and the peritubular cuff is comparatively very small or nonexistent (Fig. $1 \mathrm{~b})$. In vitro tensile yield $\left(\sigma_{\mathrm{y}}\right)$ and ultimate tensile $\left(\sigma_{U T S}\right)$ strengths levels were measured in Hank's Balanced Salt Solution (HBSS) to be $\sigma_{\mathrm{y}} \sim 50-65 \mathrm{MPa}$ and $\sigma_{\mathrm{UTS}} \sim 70-80 \mathrm{MPa}$, respectively. Elastic moduli were obtained from sound speed measurements made along orientations perpendicular and parallel to the tubule axis; values of $\sim 13-17 \mathrm{GPa}$ for the Young's modulus, 5-6 GPa for the shear modulus were obtained, with the higher values associated along the directions perpendicular to the tubules [18].

\subsection{Fracture toughness tests}

Five different orientations were examined, as shown schematically in Fig. 2, with a total of $N=16$ test samples:

- Tubule long axis perpendicular to the notch plane (XZ) and notch direction (Y) referred to as "perpendicular" orientation (Fig. 2a) - $(N=3)$,

- Tubule long axis inclined to (nominally at $\sim 45^{\circ}$ ) to the notch plane and notch direction referred to as "inclined perpendicular" orientation (Fig. 2b) - $(N=3)$,

samples [e.g., 10], gave unrealistically high values. 
- Tubule long axis parallel to the notch plane and notch direction, referred to as "inplane parallel" orientation (Fig. 2c) - $(N=3)$,

- Tubule long axis parallel to the notch plane and perpendicular to notch direction, referred to as "anti-plane parallel" orientation (Fig. 2d) - $(N=3)$, and

- Tubule long axis parallel to the notch plane and inclined to (nominally at $\sim 45^{\circ}$ ) to notch direction, referred to as "inclined parallel" orientation (Fig. 2e) - $(N=3)$.

It should be noted here that the orientation can be difficult to define in advance as the tubules often do not take a straight path, as illustrated in Fig. 3 by the curved path of the tubules on a fracture surface for one of the "in-plane parallel" orientations. Similar observations have previously been reported for both human [4] and elephant dentin [17]. In fact, with the exception of the root, the tubules in human dentin do not run a straight course from the enamel to the pulp; rather, from the cervical margin through the crown, the tubules have a complex, S-shaped curvature [19]. In light of this, it is almost impossible to align the fracture plane precisely with the tubule axes a priori, and postfractographic observations must be used to determine the exact orientation.

For each orientation, fracture toughness tests were performed in general accordance with ASTM Standards [20]. Compact-tension, C(T), samples (Fig. 4) were used, and were machined from $15-\mathrm{mm}$ square cubes of dentin, which were first obtained through rough sectioning. After machining, specimens were wet polished up to a 1200 grit finish, followed by a final polishing step using a $1 \mu \mathrm{m}$ alumina suspension. Specimens with widths of $W \sim 3.5$ to $7 \mathrm{~mm}$ and thicknesses of $B \sim 0.75$ to $1.25 \mathrm{~mm}$ were used (Fig. 4). The initial portion of the notch was carefully machined using a slow speed saw, with the final portion being introduced using a fine blade; this yielded notch root radii of typically $\rho \sim 10$ to $20 \mu \mathrm{m}$. Samples were then fatigue precracked in room air at a nominal stressintensity range of $\sim 0.9 \mathrm{MPa} \sqrt{ } \mathrm{m}$ to a crack length to width ratio of $a / W \sim 0.45-0.55$.

The $\mathrm{C}(\mathrm{T})$ specimens were loaded to failure in vitro in Hank's Balanced Salt Solution (HBSS) at ambient temperature using an $\mathrm{ELF}^{\circledR} 3200$ series voice coil-based mechanical testing machine (EnduraTEC Inc., Minnetonka, MN). The tests were performed under displacement control with a constant cross-head movement rate of $0.02 \mathrm{~mm} / \mathrm{min}$. In accordance with the ASTM Standard E-399 for Plane-Strain Fracture Toughness testing 
[20], the fracture toughness, $K_{\mathrm{c}}$ at fracture was calculated from the load history using the expression:

$$
K_{\mathrm{c}}=\frac{P_{\mathrm{c}}}{B W^{1 / 2}} f(a / W),
$$

where $P_{\mathrm{c}}$ is the load at fracture instability, $B$ is the specimen thickness, $W$ is the specimen width, $a$ is the notch plus crack length, and $f(a / W)$ is dimensionless function of $a / W$ given by:

$$
f(a / W)=\frac{[2+(a / W)]\left[0.866+4.64(a / W)-13.32(a / W)^{2}+14.72(a / W)^{3}-5.6(a / W)^{4}\right]}{[1-(a / W)]^{3 / 2}} .
$$

According to ASTM Standard E-399, a state of plane strain is achieved when the sample thickness is greater than $2.5\left(K_{\mathrm{c}} / \sigma_{\mathrm{y}}\right)^{2}$, i.e., the thickness is significantly larger than the plastic or damage zone size of $r_{\mathrm{y}} \sim 1 / 2 \pi\left(K_{\mathrm{c}} / \sigma_{\mathrm{y}}\right)^{2}$. For elephant dentin, this would require samples thicknesses greater than approximately $0.6 \mathrm{~mm}$ to yield a plane-strain $K_{\mathrm{c}}$ value. Given that this requirement is met for the specimens used in this study, it is believed that a state of plane strain exists such that the toughness values measured with the current test specimens would represent lower-bound estimates with respect to variations in sample size.

\subsection{Fractographic observations}

Post-failure fractographic observation of the fracture surfaces of the failed $\mathrm{C}(\mathrm{T})$ specimens were made in the scanning electron microscope (SEM), operating at 6-10 kV in a conventional back-scattered electron mode. Samples were imaged after first coating with a gold-palladium alloy. In addition to providing information on the mechanisms of fracture, these observations were necessary to determine unambiguously the orientation of the fracture plane with respect to the tubules.

\subsection{Crack path observations}

To understand the mechanisms associated with how microstructural orientation affects fracture in dentin, crack path trajectories were examined. However, since fracture in 
dentin tends to be brittle in nature, with crack initiation essentially simultaneous with unstable (catastrophic) fracture, a novel double-notch four-point bend geometry was used to generate a stable crack (Fig. 5). This technique, which has been previously used by the authors to discern whether fracture is locally stress- or strain-controlled in human dentin [4], involves loading a beam with two nominally identical notches to failure under four-

point bending. Under four-point (pure) bending, both notches experience the same bending moment; thus when one notch fails, the microstructure and local cracking events immediately prior to unstable fracture are effectively "frozen-in" at the unfractured notch. Since in the presence of some degree of inelastic deformation (e.g., plastic deformation), the maximum local strains are at the notch root whereas the maximum local stresses are ahead of the notch (near the elastic/plastic interface), the location of the local cracking events prior to instability gives an indication of whether fracture is locally stress- or strain-controlled. Moreover, if loaded further, this sample geometry permits the creation of a stable crack emanating from one notch which can be used to examine crack path interactions with the salient microstructure features during fracture. In the present work, such double-notch four-point bend tests were performed in HBSS at ambient temperature in two orientations, specifically with the tubule long axes nominally perpendicular and parallel to the fracture plane. Crack paths at the surface of the specimen were subsequently imaged in the SEM using the back-scattered electron mode.

\section{Results}

\subsection{Fracture toughness results}

The results of the in vitro fracture toughness testing in the five different orientations (Fig. 2) are plotted in Fig. 6, with a typical fractured C(T) specimen shown in Fig. 7. The measured data indicate a clear trend of how the toughness of dentin varies with tubule orientation, with individual $K_{\mathrm{c}}$ values listed in Table I. It is apparent that cracking perpendicular to the tubular orientation occurs with the lowest toughness $\left(K_{\mathrm{c}} \sim 1.56\right.$ $\mathrm{MPa} \sqrt{\mathrm{m}})$ whereas cracking (in-plane) parallel to the tubules occurs at the highest $\left(K_{\mathrm{c}} \sim\right.$ 2.59 MPa $\sqrt{\mathrm{m}})$. Indeed, the "anti-plane" and "in-plane parallel" orientations are, respectively, $\sim 55 \%$ and $65 \%$ tougher than the "perpendicular" orientation. Basic $F$-test 
analysis $(5 \%$ level) of these data indicates that the perpendicular orientations are not statistically significant $\left(F_{(1,4)}=7.149\right)$, and the same is true for the three parallel orientations investigated $\left(F_{(1,6)}=3.171\right)$. However, analysis of a perpendicular orientation (say, "perpendicular") and a parallel orientation (say, "in-plane parallel") do indicate that such toughness values are significant $\left(F_{(1,4)}=176.327\right)$. For comparison, the fracture toughness measured previously for fatigue precracked human dentin in the "perpendicular" orientation was $1.79 \mathrm{MPa} \sqrt{ } \mathrm{m}$ for appropriately precracked specimens in the same orientation [14].

\subsection{Fractographic observations}

Examination of the unbroken notch in fractured double-notch samples revealed that crack initiation, specifically, the first evidence of crack formation, occurred directly at the notch root for all orientations studied. Consistent with a previous study on human dentin [4], this implies that fracture is locally strain-controlled. Scanning electron micrographs

of the final fracture surfaces are shown in Fig. 8 for the various orientations. Depending upon the orientation, the tubules appear as either voids on the fracture surface, as in Figs. $8 \mathrm{a}$ and $8 \mathrm{~b}$, or as "tire" tracks, as in Figs. 8c-e.

\subsection{Crack path observations}

SEM observations of the crack paths were made normal to the fracture surface of arrested cracks in the double-notched samples. Crack-path profiles for the "perpendicular" and "anti-parallel" orientations were specifically examined; results are described below:

- Perpendicular orientation: The crack paths for the "perpendicular" orientation are shown in Fig. 9, and can be seen to be relatively flat with some evidence of out-ofplane deflections and secondary cracks (indicated by white arrows in Fig. 9a). These deflections are the result of the crack deviating along the tubule, but the resulting asperity on the crack surface never exceeds $\sim 10$ to $20 \mu \mathrm{m}$ in height. The consequent contact between the crack surfaces during crack opening can result in some degree of toughening due to elastic bridging; however, the magnitude of this effect is likely to be small due to the minimal number of such contacts. Evidence of microcracking 
surrounding the main crack can also be seen, especially in the intertubular matrix (indicated by white arrows in Fig. 9b). Since such microcracking results in some degree of dilation in the highly stressed region close to the crack, this is another potential toughening mechanism in dentin.

- Parallel orientations: Figs. 10-11 show the corresponding crack paths for the antiparallel orientations. It is apparent that, compared to the "perpendicular" orientation (Fig. 9a), there is a smaller degree of out-of-plane deflection in the crack profiles for these orientations (Fig. 10a). Microcracking is again in evidence, and appears to originate at the tubules (indicated by white arrows in Fig. 10b), although the tubules themselves do not markedly influence the path of the crack, similar to behavior in human dentin [4]. A significant difference from the "perpendicular" orientations, however, is the occurrence of uncracked ligament bridging in the wake of the crack tip; this is indicated by the white arrows in Fig. 10a. Such "bridges", which are almost certainly "two-dimensional" in nature, appear to be formed as a consequence of the three-dimensional, non-uniform extension of the crack front or where the main crack attempts to link-up with small cracks initiated at tubules ahead of the crack tip. In addition, imaging between the crack surfaces reveals significant crack bridging by intact collagen fibrils both near (Fig. 11a,b) and well behind $(\sim 100 \mu \mathrm{m})$ the crack tip (Fig. 11c). Neither uncracked ligament nor collagen fibril bridging was observed in the less tough "perpendicular" orientation.

\section{Discussion}

\subsection{General considerations}

The results of this study confirm that the fracture toughness of dentin does indeed vary with orientation, with the highest toughness found where the crack is propagating parallel to the long axis of the tubules, and the lowest where it is perpendicular to the tubular axis. A major factor defining these phenomena in dentin and specifically the mechanisms that underpin the toughness and its anisotropy is how the crack interacts with the salient features of the microstructure. This issue has been well studied in more traditional 
materials, such as metals, ceramics and polymers, but never adequately addressed for dentin. Essentially, microstructure can influence toughness in two primary ways [21,22]:

(1) It can affect the inherent resistance to microstructural damage and fracture ahead of the crack tip, which is termed intrinsic toughening, and/or

(2) It can promote crack-tip shielding, i.e., act to reduce the stress intensity actually experienced at or behind the crack tip, which is termed extrinsic toughening.

Although crack propagation can be considered as a mutual competition between these two classes of mechanisms, intrinsic toughening tends to dominate in ductile materials, whereas extrinsic mechanisms are generally the main source of toughening in brittle materials and many composites. Given the relatively brittle nature of dentin and the nature of the crack-microstructure interactions described above, extrinsic mechanisms appear to provide the primary contributions to the toughness of dentin. Potential mechanisms are illustrated schematically in Fig. 12 and are discussed briefly below, together with theoretical calculations to estimate the relative contributions of each of these mechanisms to the fracture toughness.

\subsection{Theoretical estimates of the toughening contributions}

By considering simple models for toughening mechanisms, we can assess their relative contributions, and their variation with orientation.

- Crack deflection (Fig. 12a): Where cracks deviate from the plane of maximum driving force, e.g., maximum tensile stress, the stress intensity experienced at the crack tip is reduced, thereby providing a source of toughening. Crack deflection is promoted by features in the microstructure that deviate the crack path from this plane, e.g., by crack deflection at hard particles or the crack path having an affinity for a specific dispersed phase. For dentin in the "parallel" orientation with respect to the tubules, there was practically no out-of-plane deflection of the crack (Fig. 10a), implying that the contribution to toughening due to this mechanism is insignificant in this orientation. The same is true at the macroscopic level for the "perpendicular" orientation (Fig. 9a). However, as noted previously, the crack does make occasional small (up to $20 \mu \mathrm{m}$ ) deflections locally along the tubule axis. The local stress 
intensities at the tip of such a kink can be evaluated using crack deflection mechanics. Assuming for the sake of simplicity that these deflections/kinks represent in-plane tilts through an angle $\alpha$ to the precrack plane, then the local mode I and mode II stress intensities, $k_{1}$ and $k_{2}$, at the deflected crack tip will be given by $[23,24]$ :

$$
\begin{aligned}
& k_{1}(\alpha)=c_{11}(\alpha) K_{\mathrm{I}}+c_{12}(\alpha) K_{\mathrm{II}}, \\
& k_{2}(\alpha)=c_{21}(\alpha) K_{\mathrm{I}}+c_{22}(\alpha) K_{\mathrm{II}},
\end{aligned}
$$

where $K_{\mathrm{I}}(1.5 \mathrm{MPa} \sqrt{\mathrm{m}})$ and $K_{\mathrm{II}}(=0)$ are, respectively, the mode I and mode II global (applied) stress intensities for a main crack, and the coefficients, $c_{\mathrm{ij}}(\alpha)$, are mathematical functions of the deflection angle, $\alpha\left(\sim 90^{\circ}\right)$, given in refs. [23,24]. The effective stress intensity at the tip of the deflected crack tip, $K_{\mathrm{d}}$, can then be calculated by summing the mode I and mode II contributions in terms of the strain energy release rate, viz:

$$
K_{\mathrm{d}}=\left(k_{1}{ }^{2}+k_{2}^{2}\right)^{1 / 2},
$$

which suggests that the value of the stress intensity at the crack tip is reduced locally by some $50 \%$ due to such deflection. Whereas this appears to be a fairly significant contribution to the toughness in the "perpendicular" orientation, it should be noted that this is a short-range effect which affects the local stress intensity at the few instances where such crack-path deviations occur; overall, it will not contribute that significantly to the globally measured toughness in this orientation.

- Crack bridging (Fig. 12b,c): Crack bridging is the commonest form of crack-tip shielding, particularly in fiber composites where intact fibers tend to bridge the crack and oppose crack opening. In the present case, crack-path observations did indicate possible bridging by collagen fibers (Fig. 11), akin to that proposed for bone [25]. Furthermore, it is feasible that bridging also occurs where the dominant crack links with smaller cracks ahead of the crack tip to form uncracked ligaments (Fig. 10a). These mechanisms, however, are only observed in the "parallel" orientation in dentin. Theoretical estimates of contribution to toughening from these various forms of bridging can be made using existing models in the literature. For toughening associated with bridging by the collagen fibrils, the uniform traction Dugdale zone 
model from ref. [26] can be employed to obtain an estimate of the resulting decrease in the stress intensity, $K_{\mathrm{b}}{ }_{\mathrm{f}}$, viz:

$$
K_{\mathrm{b}}{ }^{\mathrm{f}}=2 \sigma_{\mathrm{b}} f_{\mathrm{f}}\left(2 l_{\mathrm{f}} / \pi\right)^{-1 / 2}
$$

where $\sigma_{\mathrm{b}}$ is the normal bridging stress on the fibrils $(\sim 100 \mathrm{MPa}), f_{\mathrm{f}}$ is the effective area fraction of the collagen fibrils active on the crack plane $(\leq 0.2$, from crack path observations), and $l_{\mathrm{f}}$ is the bridging zone length $(\leq 10 \mu \mathrm{m}$, from crack path observations). Using estimates of these parameters, a value of $K_{\mathrm{b}}{ }^{\mathrm{f}}<0.10 \mathrm{MPa} \sqrt{\mathrm{m}}$ was obtained.

For the uncracked ligament bridging, a limiting crack-opening displacement approach [27] can be used to obtain the contribution from this bridging mechanism, $K_{\mathrm{b}}^{\mathrm{ul}}$, to the toughness in the "parallel" orientation,

$$
K_{\mathrm{b}}^{\mathrm{ul}}=-f_{\mathrm{ul}} K_{\mathrm{I}}\left[\left(1+l_{\mathrm{ul}} / r b\right)^{1 / 2}-1\right] /\left[1-f_{\mathrm{ul}}+f_{\mathrm{ul}}\left(1+l_{\mathrm{ul}} / r b\right)^{1 / 2}\right]
$$

where $f_{\mathrm{ul}}$ is the area fraction of bridging ligaments on the crack plane $(\sim 0.2-0.4$, from crack path observations), $K_{\mathrm{I}}$ is the applied (far-field) stress intensity $(2.4 \mathrm{MPa} \sqrt{\mathrm{m}}), l_{\mathrm{ul}}$ is the bridging zone size ( $\sim 50-300 \mu \mathrm{m}$, from crack path observations), $r$ is a rotational factor $(0.195-0.470$ [27]) and $b$ is the length of the remaining uncracked region ahead of the crack. Again, substituting typical values for these parameters, toughening of the order of $K_{\mathrm{b}}{ }^{\mathrm{ul}} \sim 0.10$ to $0.44 \mathrm{MPa} \sqrt{\mathrm{m}}$ can be expected.

- Damage-zone microcracking (Fig. 12d): Although the formation of microcracks in the damage zone ahead of the crack tip can often act to lower the intrinsic toughness, the consequent formation of a microcracking zone in the wake of the crack and the resultant dilation and reduction in modulus in this zone (which is constrained by the surrounding rigid material) can act to shield the crack tip and hence, extrinsically toughen the material. This mechanism has been widely associated with toughening in bone [e.g., 38-30], and has been reported previously for human dentin [4]. At steadystate, the increase in toughness can be estimated in terms of the closure stress intensity for such dilatational toughening $[29,30]$ and the modulus reduction $[31,32]$ by:

$$
K_{\mathrm{mic}}=0.22 \varepsilon_{\mathrm{m}} E^{\prime} f_{\mathrm{m}} l_{\mathrm{m}}^{1 / 2}+\beta f_{\mathrm{m}} K_{\mathrm{c}}
$$


where $\varepsilon_{\mathrm{m}}$ is the residual volumetric strain $(\sim 0.06), E^{\prime}$ is the plane-strain elastic modulus, $f_{\mathrm{m}}$ is the effective volume fraction of microcracks, $l_{\mathrm{m}}$ is the size (specifically height) of the microcrack zone (estimated to be $\sim 15$ to $37 \mu \mathrm{m}$, depending on tubule orientation), $\beta$ is a parameter dependent on the Poisson's ratio $\left(\sim 1.2\right.$ [32]) and $K_{\mathrm{c}}$ is the matrix toughness for dentin $(\sim 1.5 \mathrm{MPa} \vee \mathrm{m})$. Using Eq. 7, the contribution to the fracture toughness of dentin from extrinsic shielding due to microcracking can be estimated to be on the order of $0.25 \mathrm{MPa} \sqrt{\mathrm{m}}$ in the "perpendicular" orientation and $0.3 \mathrm{MPa} \sqrt{\mathrm{m}}$ in the "parallel" orientation.

It should be appreciated that these estimates are only approximate. Moreover, numerically they represent only small stress-intensity values. However, as the intrinsic toughness of dentin is only on the order of 1-2 MPa $\sqrt{\mathrm{m}}$, extrinsic toughening contributions from bridging ( 0.2 to $0.5 \mathrm{MPa} \sqrt{\mathrm{m}})$ and microcracking $(\sim 0.3 \mathrm{MPa} \sqrt{\mathrm{m}})$ are nevertheless significant. Furthermore, as discussed below, as the difference in toughness between the "perpendicular" and "parallel" orientations in dentin is only $\sim 1 \mathrm{MPa} \sqrt{\mathrm{m}}$, bridging by collagen fibrils $(\sim 0.1 \mathrm{MPa} \sqrt{\mathrm{m}})$ and by uncracked ligaments $(\sim 0.1$ to 0.4 $\mathrm{MPa} \sqrt{\mathrm{m}}$ ) in the latter orientation can play a substantial role in explaining the toughness anisotropy.

\subsection{Implications}

It has been suggested that the microstructure of dentin can be considered as a cylindrical fiber reinforced composite [3], with the matrix as intertubular dentin and the reinforcements as the tubule lumens and the concomitant peritubular dentin cuffs. However, with such a depiction, the optimum toughness would be expected for crack extension perpendicular to the "fibers", whereas the experimental results (Fig. 6) reveal exactly the opposite trend, with the "perpendicular" orientation having the lowest toughness and the (in-plane) "parallel" orientation, the highest. This contradiction clearly suggests that the tubules do not play a direct role in affecting the fracture toughness of dentin. Indeed, this conclusion is consistent with observations that the elastic properties in dentin are dominated by the intertubular matrix, rather than the tubules themselves 
[12], and with direct observations of crack trajectories, both in the present work and in human dentin [4], which show only minimal interaction between the crack and the tubular voids (Figs. 9a, 10a).

Since the microcracking density appears to be roughly comparable in all orientations (as supported by the calculations for toughening due to microcracking described above), an alternative explanation for the effect of tubule orientation on the toughness of dentin, however, can be related to two other factors: (i) the orientation of the collagen fibers, and (ii) the creation of uncracked ligaments. The type I collagen fibril network forms the framework of dentin and, except very close to the dentin-enamel junction, these fibrils are oriented in layers perpendicular to the dentinal tubules [2]. Indeed, should these fibrils, by remaining unbroken in the crack wake, act to bridge the crack, it is reasonable to expect that this would lead to a higher toughness in the "parallel" orientations. Support for this notion is provided by the micrographs of crack paths in Fig. 11 which clearly show collagen fibrils spanning the crack, although the potency of this mechanism still awaits experimental verification ${ }^{2}$. However, the calculations reported above do suggest that bridging by uncracked ligaments may be the more important contribution to toughening in the "parallel" orientations. Here, smaller cracks that form at tubules ahead of the crack tip, in the process of linking with the main crack, can leave two-dimensional uncracked regions that can act as more macroscopic bridges (Figs. 11a); they can also be formed by the nonuniformity of crack advance along the crack front in three dimensions. There is strong evidence for both these mechanisms in the "parallel" orientations - neither can occur in the "perpendicular" orientation - and their contribution $(\sim 0.2$ to 0.5 $\mathrm{MPa} \sqrt{\mathrm{m}}$ ) does appear to be significant, and can explain the magnitude of the anisotropy in the fracture toughness in dentin.

We therefore conclude that there are several significant extrinsic toughening mechanisms acting in concert that contribute to the fracture toughness of dentin, specifically from crack deflection (and perhaps elastic bridging), microcracking, and crack bridging by collagen fibrils and uncracked ligaments, all processes that result from the interaction of the crack path with the microstructure of dentin. Whereas some degree

\footnotetext{
${ }^{2}$ This can be achieved by comparing the crack-opening displacement with that of a traction-free crack (see ref. [33]); such experiments using in situ SEM measurements are currently in progress.
} 
of toughening may result from crack deflection that can occur in the "perpendicular" orientation due to minimal crack path excursions associated with interactions between the main crack and small cracks associated with the dentinal tubules, this contribution is likely to be minimal as it only affects the local stress intensity in a relatively few isolated regions. The principal toughening mechanisms appear to be crack bridging $(\sim 0.2$ to 0.5 $\mathrm{MPa} \sqrt{\mathrm{m}})$ and microcracking $(\sim 0.3 \mathrm{MPa} \sqrt{\mathrm{m}})$. Since the latter contribution does not vary much with orientation, we conclude that the origin of the anisotropy in the fracture toughness of dentin, where $K_{\mathrm{c}}$ values in elephant dentin were found to be some 55 to $65 \%$ higher for cracks propagating in the plane parallel, as compared to perpendicular, to the tubular axis, can be related to crack bridging. Specifically, bridging by the collagen fibrils $(\sim 0.1 \mathrm{MPa} \sqrt{\mathrm{m}})$ and more importantly by uncracked ligaments $(\sim 0.1$ to 0.4 $\mathrm{MPa} \sqrt{ } \mathrm{m})$, created just before linkage of dentinal tubular cracks with the main crack tip, are postulated to provide the main source of the higher toughness in the "parallel" orientations.

\subsection{Relevance to bone}

This study has applicability in the broader context of mineralized tissues like bone, as dentin has similar composition but lacks the complicated microstructure and remodeling processes of bone. This allows for the isolation of the contribution to toughening from an aligned collagen scaffold. Indeed, these advantages have been exploited by Currey et al. [15] who used Narwhal tusk dentin to elucidate the effects of collagen fibril alignment on the elastic properties of bone. Although bone has a more complex microstructure than dentin [34], at the shortest length scale it is composed, like dentin, of type-I collagen fibrils bound and impregnated with carbonated apatite nanocrystals. These fibrils are further organized at a microstructural length scale in bone into a lamellar structure with roughly orthogonal orientations of adjacent lamellae. Permeating this lamellar structure are the osteons: large vascular channels surrounded by circumferential lamellar rings. Although various toughening mechanisms have been proposed for bone, the difficulty clearly lies in determining the relative importance of each of these microstructural hierarchies on crack initiation, subsequent crack propagation and consequent fracture. At 
long length scales, the generation of microcracks in advance of the crack tip is believed to contribute to the fracture toughness, specifically via crack-tip shielding [e.g., 28,35,36], and cement lines and osteons are believed to affect the energetics of crack propagation [37]. More recently, the role of the mineralized collagen fibril has been explored [38,39], and a fiber-bridging model has been proposed as a toughening mechanism [25]. However, as with dentin, the existence of crack bridging, and its possible effect on fracture toughness, has been based largely on inference, since the heterogeneous microstructure of bone introduces many confounding variables into the proper interpretation of fracture mechanisms. Consequently, the results obtained in the present study may be useful steps for the understanding of fracture in bone, and in estimating the contribution of the mineralized fibrils to the fracture toughness.

\section{Conclusions}

Based on an experimental evaluation of the anisotropy in the in vitro fracture toughness of dentin (obtained from elephant tusks) with orientation, coupled with theoretical considerations of the contributions from the proposed toughening mechanisms, the following conclusions can be drawn:

1. Fracture toughness $K_{\mathrm{c}}$ values, determined from fatigue precracked compact-tension samples, were found to be $\sim 55-65 \%$ higher for cracks propagating parallel to the long axis of the dentinal tubes compared to crack propagation perpendicular to the tubules. Specifically, for the "in-plane" and "anti-plane parallel" orientations, $K_{\mathrm{c}}$ values were between 2.4 MPa $\sqrt{\mathrm{m}}$ and $2.6 \mathrm{MPa} \sqrt{\mathrm{m}}$, respectively, compared to $1.6 \mathrm{MPa} \sqrt{\mathrm{m}}$ for the "perpendicular" orientation. In all orientations, fracture was found to be locally strain-controlled.

2. Although there was evidence of microcracking in all orientations, the salient distinction between the "parallel" and "perpendicular" orientations was the occurrence, in the "parallel" orientations, of crack bridging by intact collagen fibrils and, more importantly, by uncracked ligaments, caused by the main crack trying to link with smaller cracks initiated at dentinal tubules ahead of the crack tip. Such bridging mechanisms were not observed in the "perpendicular" orientation and are 
considered to be the primary reason for the observed anisotropy in the fracture toughness of dentin.

3. Theoretical estimates of the individual effect of these extrinsic toughening mechanisms were consistent with this conclusion that the orientation-dependent anisotropy in toughness is primarily the result of crack bridging. These estimates suggest contributions of the order of $0.3 \mathrm{MPa} \sqrt{\mathrm{m}}$ due to microcracking (in all orientations), and $\sim 0.1 \mathrm{MPa} \sqrt{\mathrm{m}}$ and $\sim 0.1$ to $0.4 \mathrm{MPa} \sqrt{\mathrm{m}}$, respectively, from collagen fibril and uncracked ligament bridging (in the "parallel" orientations).

\section{Acknowledgments}

This work was supported in part by the National Institutes of Health, National Institute for Dental and Craniofacial Research under Grant No. P01DE09859. The authors wish to thank the Oakland Zoo, Oakland, CA, for supplying the elephant tusk, Prof. J. D. Currey, of the University of York, U.K., for valuable discussions on comparative anatomy, Profs. S. J. Marshall and G. W. Marshall, of the Department of Preventive and Restorative Dental Sciences, University of California, San Francisco, CA, for their support, and Drs. A. P. Tomsia, J. J. Kruzic and V. Imbeni, of the Materials Science Division, Lawrence Berkeley National Laboratory, Berkeley, CA, for experimental assistance.

\section{References}

1. Marshall GW, Marshall SJ, Kinney JH, Balooch M. The dentin substrate: Structure and properties related to bonding. J Dent 1997;25:441-458.

2. Jones SJ, Boyde A. Ultrastructure of dentin and dentinogenesis. In: Linde. editor. Dentin and dentinogenesis. Boca Raton, FL: CRC Press; 1984. 1(of 2):p 81-134.

3. Kinney JH, Marshall SJ, Marshall GW. The mechanical properties of human dentin: A critical review and reevaluation of the dental literature. Crit Reviews Oral Biol Medicine 2002; in press.

4. Nalla RK, Kinney JH, Ritchie RO. On the fracture of human dentin: Is it stress- or strain-controlled? J Biomed Mater Res 2002; in review.

5. Craig RG, Peyton FA. Elastic and mechanical properties of human dentin. J Dent Res 1958;37:710-718.

6. Lehman ML. Tensile strength of human dentin. J Dent Res 1967;46:197-201. 
7. Renson CE, Braden M. Experimental determination of rigidity modulus, Poisson's ratio and elastic limit in shear of human dentin. Arch Oral Biol 1975;20:43-47.

8. Rasmussen ST, Patchin RE, Scott DB, Heuer AH. Fracture properties of human enamel and dentin. J Dent Res 1976;55:154-164.

9. Rasmussen ST, Patchin RE. Fracture properties of human enamel and dentin in an aqueous environment. J Dent Res 1984;63:1362-1368.

10. el Mowafy OM, Watts DC. Fracture toughness of human dentin. J Dent Res 1986;65:677-681.

11. Kinney JH, Balooch M, Marshall SJ, Marshall GW, Weihs TP. Hardness and Young's modulus of human peritubular and intertubular dentin. Arch Oral Biol 1996;41:9-13.

12. Kinney JH, Balooch M, Marshall WJ, Marshall SJ. A micromechanics model of the elastic properties of human dentine. Arch Oral Biol 1999;44:813-822.

13. Iwamoto N, Ruse ND. NTP specimen fracture toughness test applied to human dentin. In: Proc. SEM Annual Conf. and Exposition on Experimental and Applied Mechanics. Portland OR; 2001. p. 90-91.

14. Imbeni V, Nalla RK, Bosi C, Kinney JH, Ritchie RO. On the in vitro fracture toughness of human dentin. J Biomed Mater Res 2002; in press.

15. Currey JD, Brear K, Zioupos P. Dependence of mechanical properties on fibre angle in narwhal tusk, a highly oriented biological composite. J Biomech 1994;27:885-897.

16. Knott JF. Fundamentals of fracture mechanics. London, U.K.: Butterworth \& Co. (Publishers) Ltd; 1976.

17. Raubenheimer EJ, Dauth J, Dreyer MJ, Smith PD, Turner ML. Structure and composition of ivory of the African elephant (Loxodonta africana). South African J Sci, 1990;86:192-193.

18. Kinney JH. University of California at San Francisco, unpublished research; 2002.

19. Ten Cate AR. Oral Histology-Development, Structure and Function. 4th edition, St. Louis, MO: Mosby (Publishers) 1994; 173.

20. ASTM E 399-90 (Reapproved 1997), Annual Book of ASTM Standards, Vol. 03.01: Metals- Mechanical testing; Elevated and low-temperature tests; Metallography, West Conshohocken, PA: ASTM; 2001.

21. Ritchie, RO. Mechanisms of fatigue crack propagation in metals, ceramics and composites: Role of crack-tip shielding. Mater Sci Eng 1988;103:15-28. 
22. Ritchie, RO. Mechanisms of fatigue-crack propagation in ductile and brittle solids. Int J Fract 1999;100:55-83.

23. Bilby BA, Cardew GE, Howard IC. Stress intensity factors at the tips of kinked and forked cracks. In: Taplin DMR. editor. Fracture 1977. Oxford, U.K.: Pergamon Press; 1978. 3:p 197-200.

24. Cotterell B, Rice JR. Slightly curved or kinked cracks. Int J Fract 1980;16:155-69.

25. Yeni YN, Fyhrie DP. Collagen-bridged microcrack model for cortical bone tensile strength. In: Proc Bioengineering Conference BED. New York, NY: ASME; 2001. 50:p 293-294.

26. Evans AG, McMeeking RM. On the toughening of ceramics by strong reinforcements. Acta Metall 1986;34:2435-2441.

27. Shang JH, Ritchie RO. Crack bridging by uncracked ligaments during fatigue-crack growth in SiC-reinforced aluminum-alloy composites. Metall Trans A 1989;20A:897908.

28. Vashishth D, Tanner KE, Bonfield W. Contribution, development and morphology of microcracking in cortical bone during crack propagation. J Biomech 2000;33:1169-1174.

29. Evans AG, Fu Y. Some effects of microcracks on the mechanical properties of brittle solids II: Microcrack toughening. Acta Metall 1985;33:1525-1531.

30. Liu G, Zhu D, Shang JK. Enhanced fatigue crack growth resistance at elevated temperature in TiC/Ti-6Al-4V composite: Microcrack-induced crack closure. Metall Mater Trans A 1995;26A:159-166.

31. Hutchinson JW. Crack tip shielding by micro-cracking in brittle solids. Acta Metall 1987;35:1605-1619.

32. Sigl LS. Microcrack toughening in brittle materials containing weak and strong interfaces. Acta Metall 1996;44:3599-3609.

33. Gilbert CJ, Ritchie RO. On the quantification of bridging tractions during subcritical crack growth under monotonic and cyclic fatigue loading in grain-bridging silicon carbide ceramic. Acta Mater 1998;46:609-616.

34. Rho JY, Kuhn-Spearing L, Zioupos P. Mechanical properties and the hierarchical structure of bone. Med Eng Phys 1998;20:92-102.

35. Vashishth D, Koontz J, Qiu S, Lundin-Cannon D, Yeni Y, Schaffler M, Fyhrie D. In vivo diffuse damage in human vertebral trabecular bone. Bone 2000;26:147-152. 
36. Parsamian GP, Norman TL. Diffuse damage accumulation in the fracture process zone of human cortical bone specimens and its influence on fracture toughness. J Mater Sci: Mater Med 2001;12:779-783.

37. Yeni YN, Norman TL. Calculation of porosity and osteonal cement line effects on the effective fracture toughness of cortical bone in longitudinal crack growth. J Biomed Mater Res 2000;51:504-509.

38. Wang X, Bank RA, Tekoppele JM, Agrawal CM. The role of collagen in determining bone mechanical properties. J Orthopaed Res 2001;19:1021-1026.

39. Wang X, Shen X, Li X, Agrawal CM. Age-related changes in the collagen network and the toughness of bone. Bone 2002;31:1-7. 


\section{List of Figures}

Fig. 1: Scanning electron micrographs of (a) the typical microstructure of elephant tusk dentin used in this study (in the plane perpendicular to the long axis of the tubules), and (b) a tubule. Note in comparison to human dentin, the slightly elliptical shape of the tubule and the lack of a mineralized peritubular dentin cuff.

Fig. 2: Schematics of the five orientations used in this investigation. The reference coordinate system with the crack in the XZ plane and the direction of nominal crack propagation in the $\mathrm{X}$ direction are also shown.

Fig. 3: A scanning electron micrograph of a fracture surface obtained for a "parallel" orientation specimen. Clearly, the tubules do not run in a straight course, but take a rather complex curved path. The direction of nominal crack growth is shown.

Fig. 4: (a) A schematic illustration of the compact-tension specimen geometry used in the present study. Typically, $a / W$ ratios of $\sim 0.45-0.55$ were utilized. Also shown is the reference coordinate axis system used for describing the orientation of the specimens. (b) An optical photomicrograph of a typical specimen is given.

Fig. 5: (a) Schematic illustration of the double-notched four-point bend test used. The crack in the region below the unbroken notch (as indicated in (b)) was examined for crack-microstructure interactions.

Fig. 6: The average fracture toughness values for the five tubule orientations investigated are plotted. The half-error bars indicate one standard deviation; the numbers are the average toughness values.

Fig. 7: A low magnification scanning electron micrograph of a fractured compact tension specimen. The notch and the final fracture surface are evident.

Fig. 8: Scanning electron micrographs of the fracture surfaces obtained for the various orientations in this study. The inset schematic indicates the orientation used. The direction of nominal crack growth is shown by the black arrow. Note that the tubules appear as voids for the "perpendicular" orientations and as "tire" tracks for the "parallel" orientations.

Fig. 9: Scanning electron micrographs of typical crack paths for the nominally "perpendicular" orientation in the context of crack-microstructure interactions. The white arrows indicate in (a) the slight out-of-plane deflections made by the crack, and in (b) microdamage in the form of microcracks, a possible toughening mechanism. 
Fig. 10: Scanning electron micrographs of typical crack paths for the nominally "antiplane parallel" orientation in the context of crack-microstructure interactions. The white arrows indicate in (a) uncracked ligament bridging, and in (b) microcracks in the vicinity of the crack.

Fig. 11: Scanning electron micrographs of typical crack paths for the nominally "antiplane parallel" orientation in the context of crack-microstructure interactions, indicating bridging by collagen fibers. Note in (b) the broken (indicated by white arrows) and unbroken fiber "bridges".

Fig. 12: Schematic illustrations of some of the possible toughening mechanisms in dentin: (a) crack deflection, (b) crack bridging (by collagen fibers), (c) uncracked ligament bridging, and (d) microcracking. 
Table I: Measured fracture toughness values as a function of tubule orientation

\begin{tabular}{lcc}
\hline Specimen Type & $\begin{array}{c}\text { Fracture toughness, } K_{\mathrm{c}} \\
(\mathrm{MPa} \sqrt{\mathrm{m}})\end{array}$ & $\begin{array}{c}\text { Average }(\mathrm{Std} . \mathrm{dvn} .) \\
(\mathrm{MPa} \sqrt{\mathrm{m}})\end{array}$ \\
\hline Perpendicular & 1.44 & $1.56(0.08)$ \\
Perpendicular & 1.63 & \\
Perpendicular & 1.60 & $1.84(0.12)$ \\
Inclined Perpendicular & 2.01 & \\
Inclined Perpendicular & 1.80 & \\
Inclined Perpendicular & 1.72 & $2.59(0.07)$ \\
In-plane Parallel & 2.65 & \\
In-plane Parallel & 2.49 & $2.44(0.11)$ \\
In-plane Parallel & 2.63 & \\
Anti-plane Parallel & 2.54 & \\
Anti-plane Parallel & 2.29 & $2.50(0.07)$ \\
Anti-plane Parallel & 2.49 & \\
Inclined Parallel & 2.58 & \\
Inclined Parallel & 2.50 & \\
Inclined Parallel & 2.41 & \\
\hline
\end{tabular}



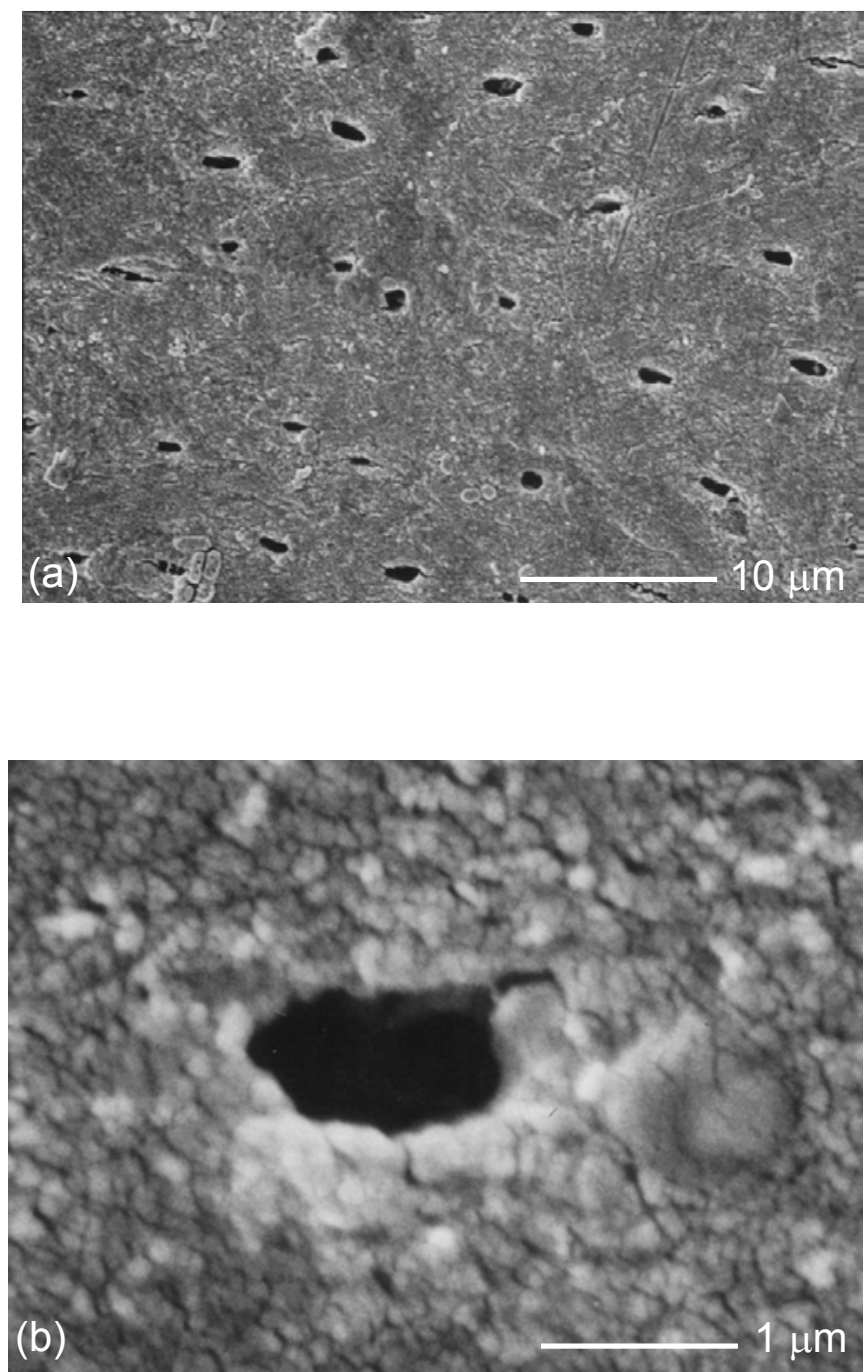

Fig. 1: Scanning electron micrographs of (a) the typical microstructure of elephant tusk dentin used in this study (in the plane perpendicular to the long axis of the tubules), and (b) a tubule. Note in comparison to human dentin, the slightly elliptical shape of the tubule and the lack of a mineralized peritubular dentin cuff. 


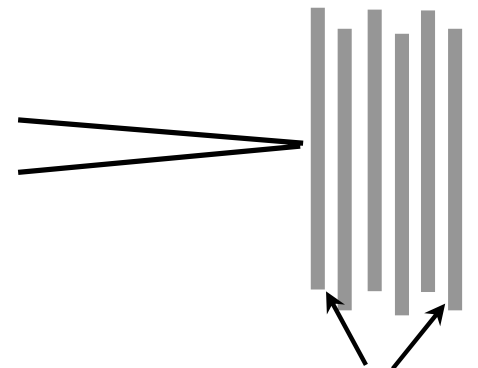

tubules

(a) Perpendicular

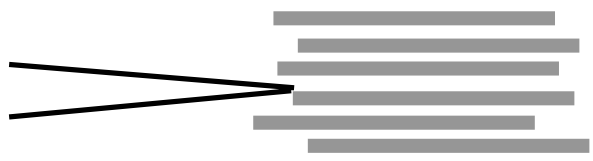

(c) In-plane Parallel

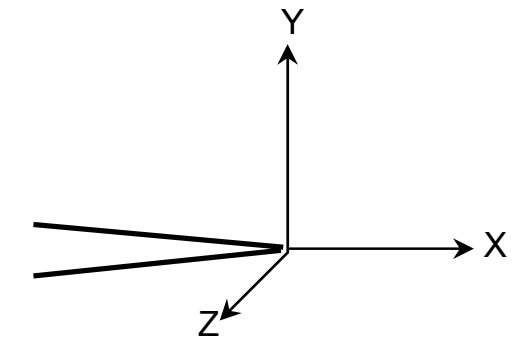

nominal crack propagation direction

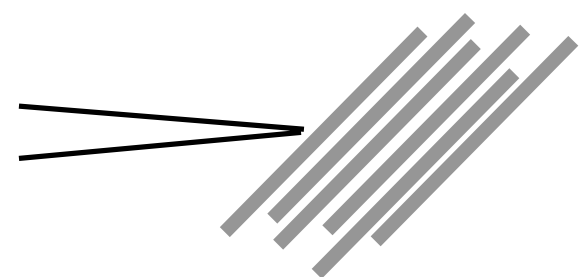

(b) Inclined Perpendicular

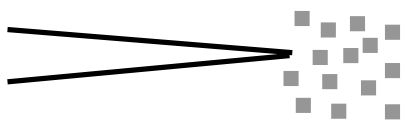

(d) Anti-plane Parallel

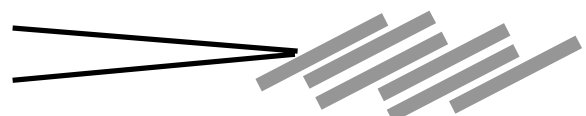

(e) Inclined Parallel

Fig. 2: Schematics of the five orientations used in this investigation. The reference coordinate system with the crack in the $X Z$ plane and the direction of nominal crack propagation in the $\mathrm{X}$ direction are also shown. 


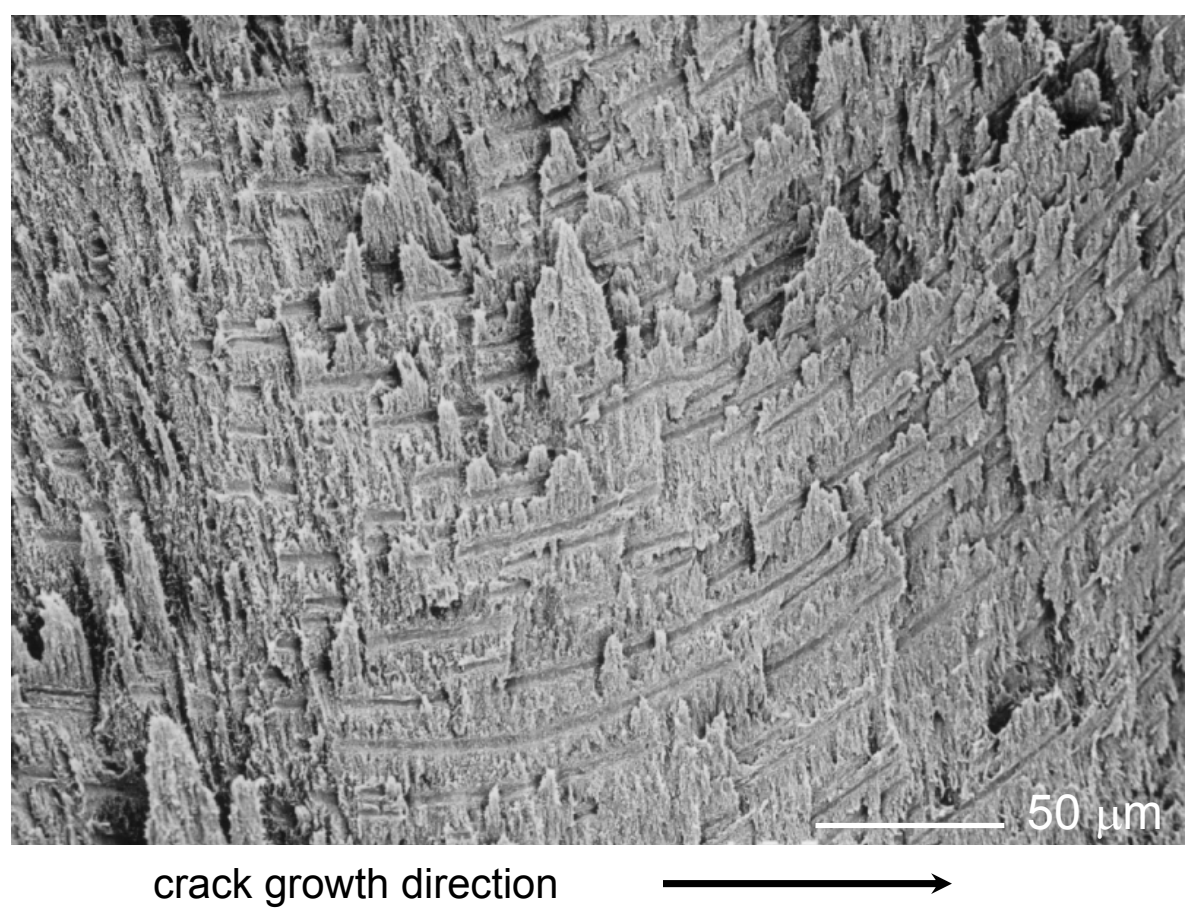

Fig. 3: A scanning electron micrograph of a fracture surface obtained for a "parallel" orientation specimen. Clearly, the tubules do not run in a straight course, but take a rather complex curved path. The direction of nominal crack growth is shown. 


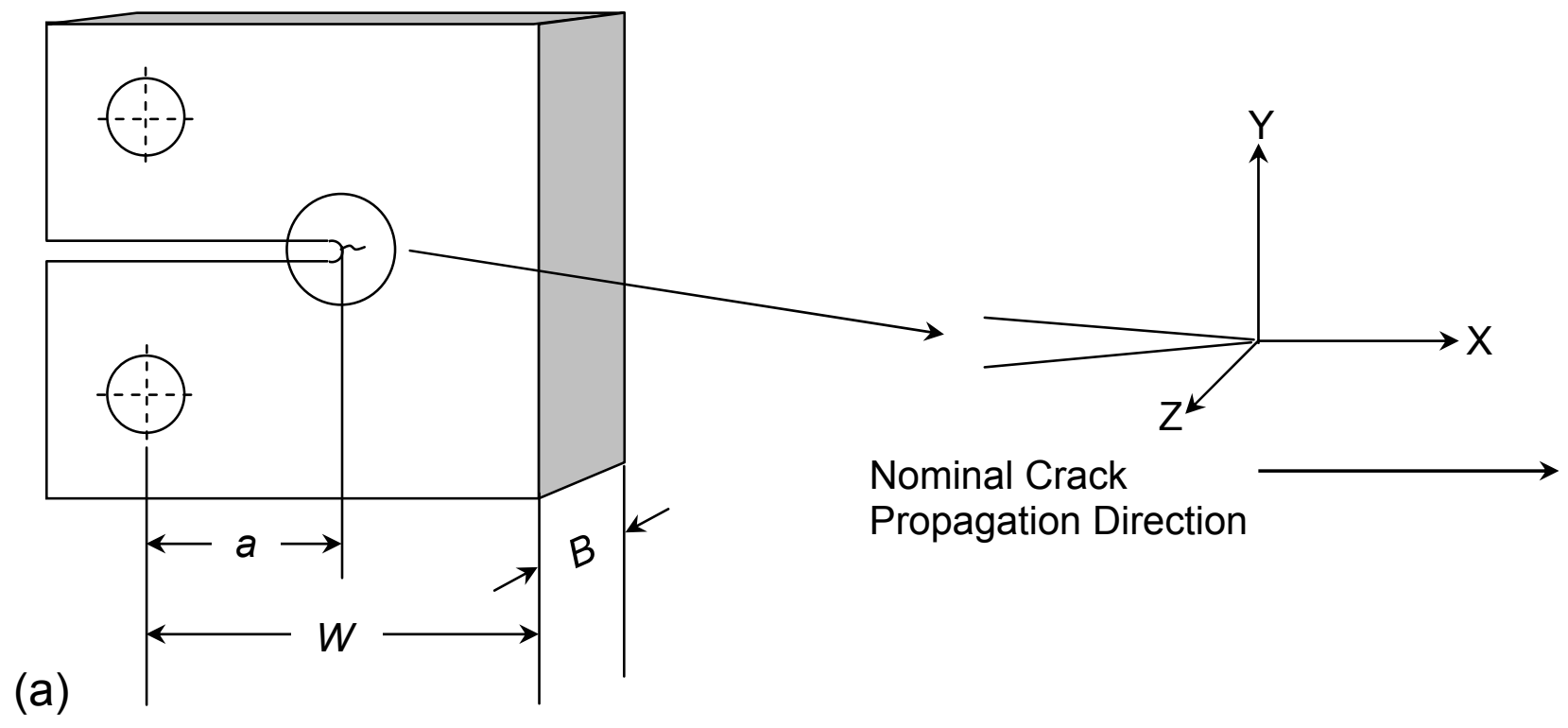

(b)

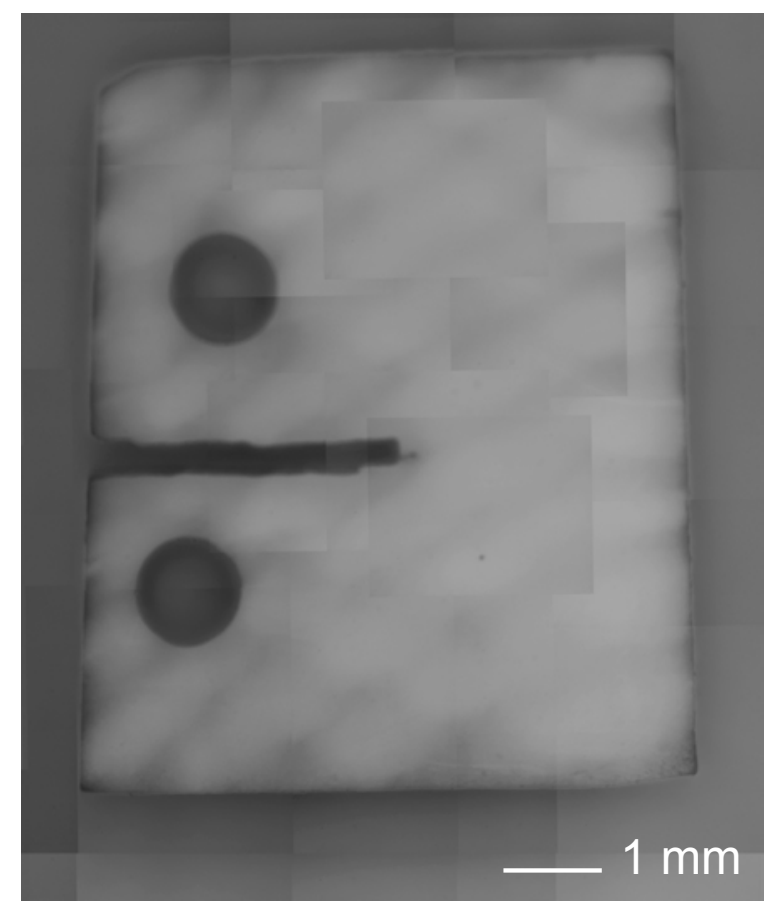

Fig. 4: (a) A schematic illustration of the compact-tension specimen geometry used in the present study. Typically, $a / W$ ratios of $\sim 0.45-0.55$ were utilized. Also shown is the reference coordinate axis system used for describing the orientation of the specimens. (b) An optical photomicrograph of a typical specimen is given. 


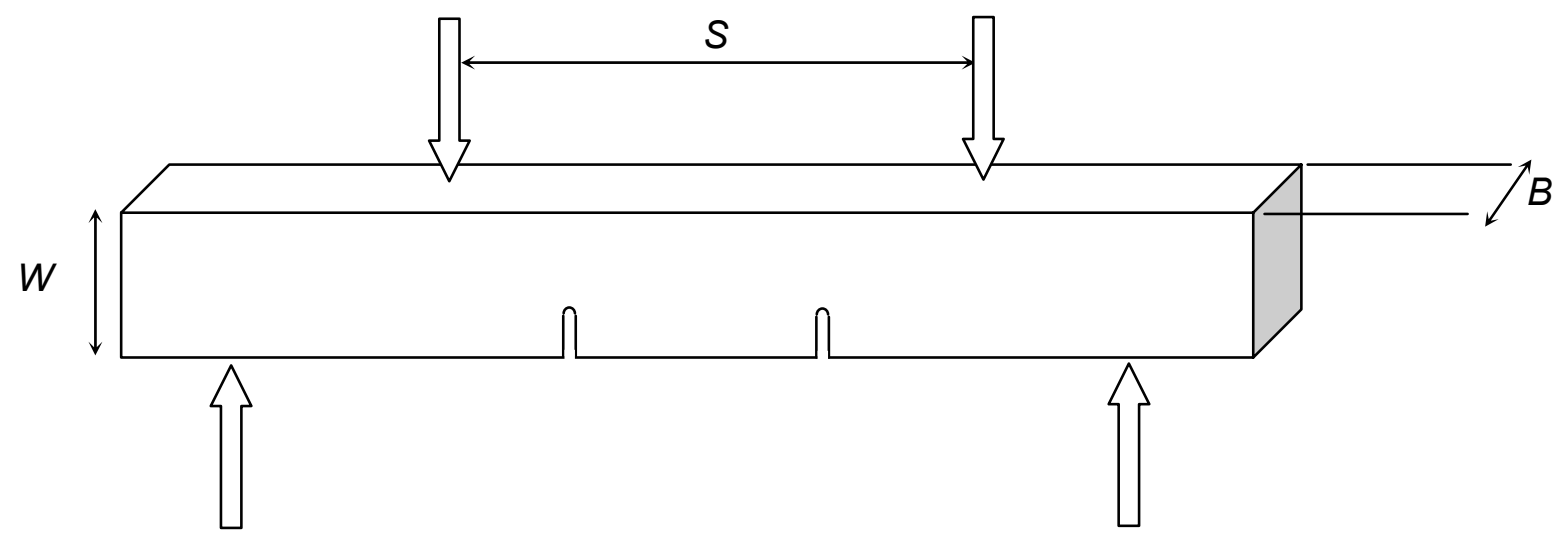

(a)

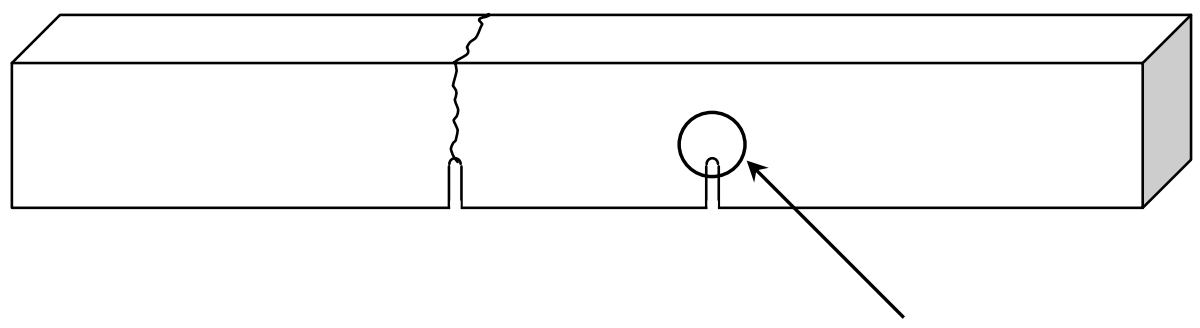

(b)

Fig. 5: (a) Schematic illustration of the double-notched four-point bend test used. The crack in the region beneath this unbroken notch (as indicated in (b)) was examined for crack-microstructure interaction. 


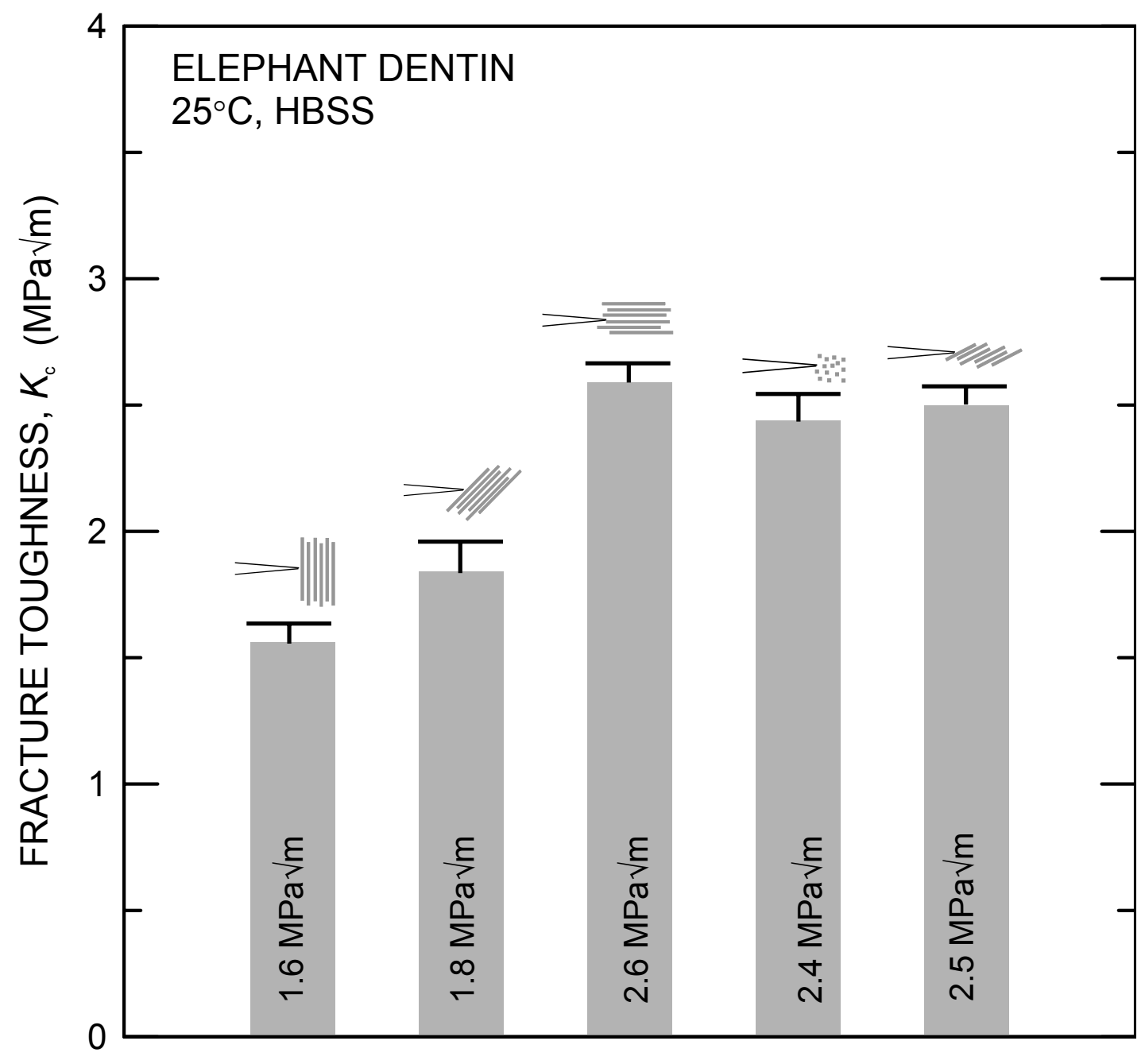

Fig. 6: The average fracture toughness values for the five tubule orientations investigated are plotted. The half-error bars indicate one standard deviation; the numbers are the average toughness values. 


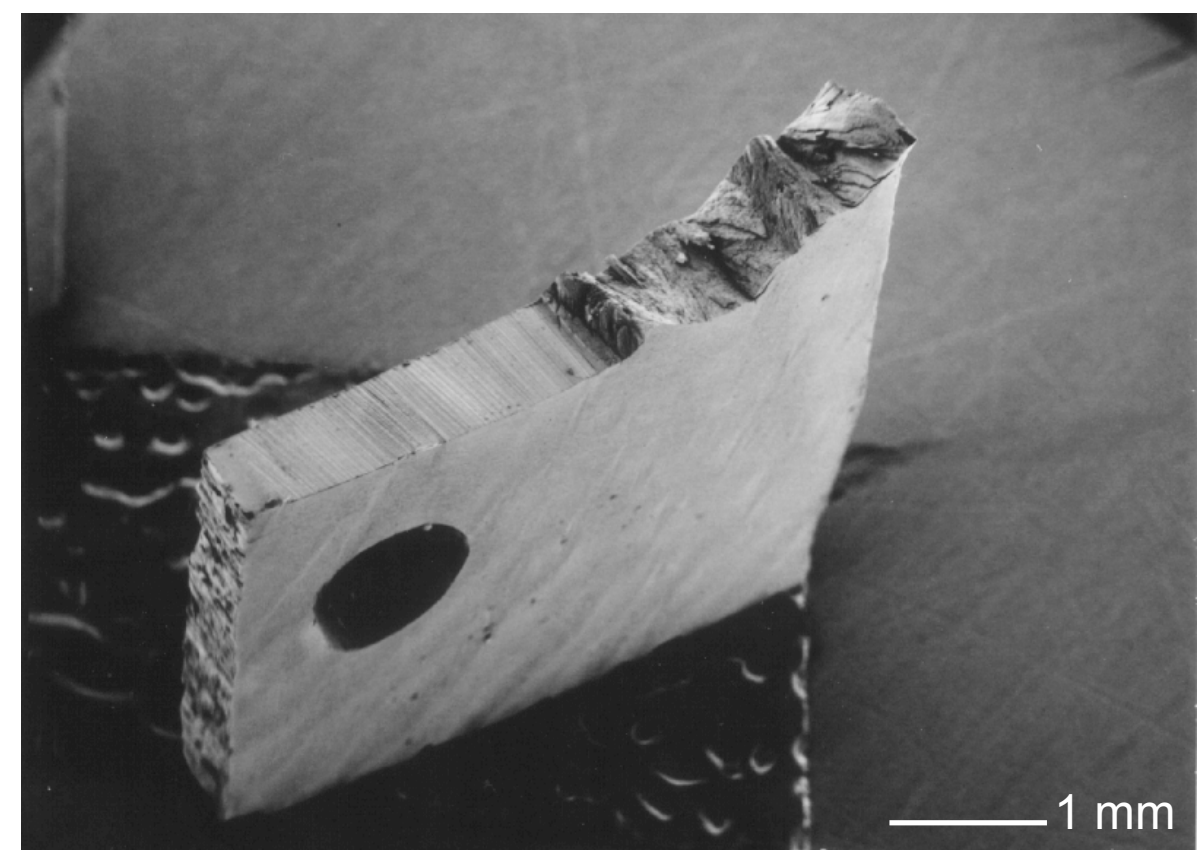

Fig. 7: A low magnification scanning electron micrograph of a fractured compact tension specimen. The notch and the final fracture surface are evident. 

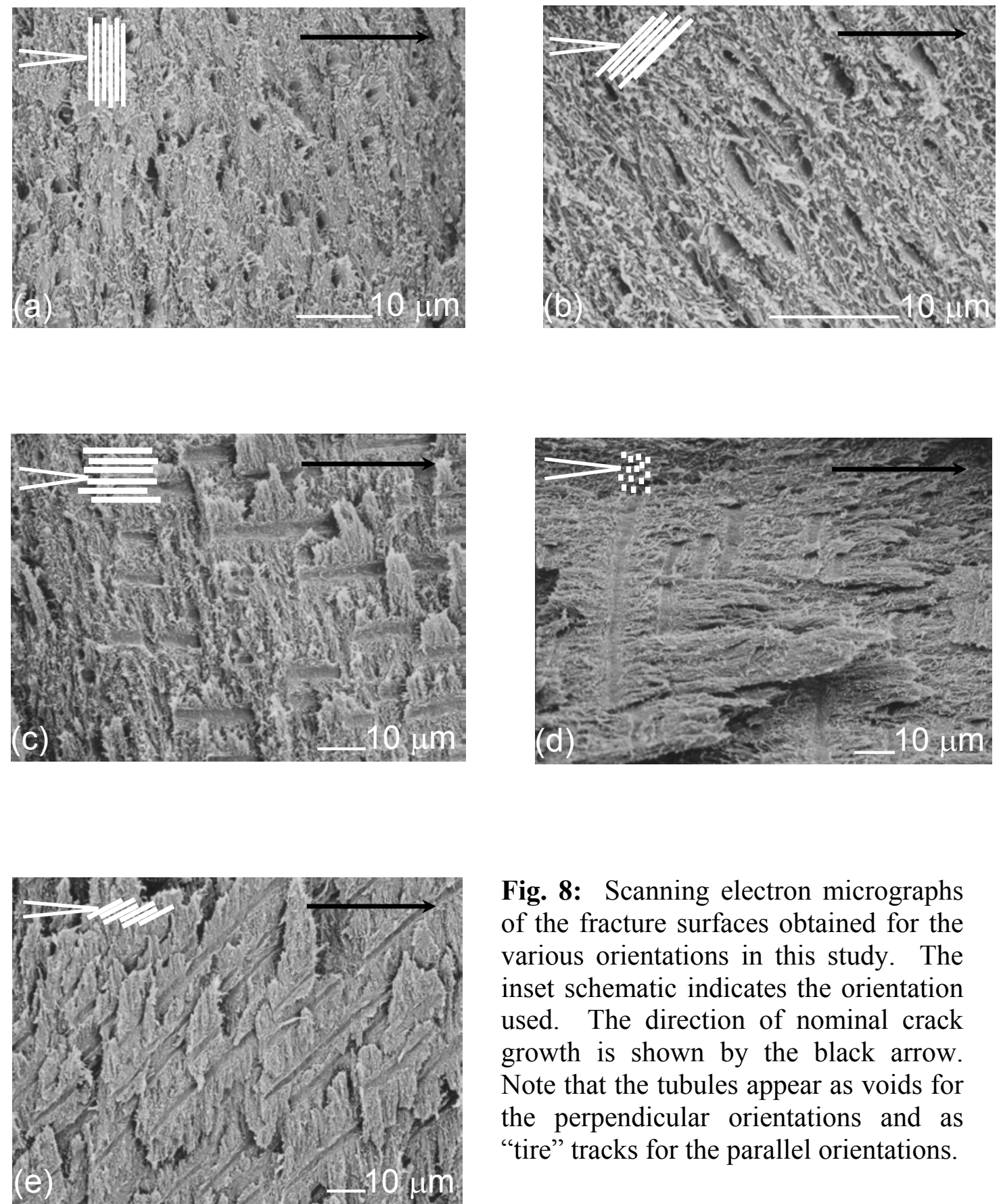

Fig. 8: Scanning electron micrographs of the fracture surfaces obtained for the various orientations in this study. The inset schematic indicates the orientation used. The direction of nominal crack growth is shown by the black arrow. Note that the tubules appear as voids for the perpendicular orientations and as "tire" tracks for the parallel orientations. 

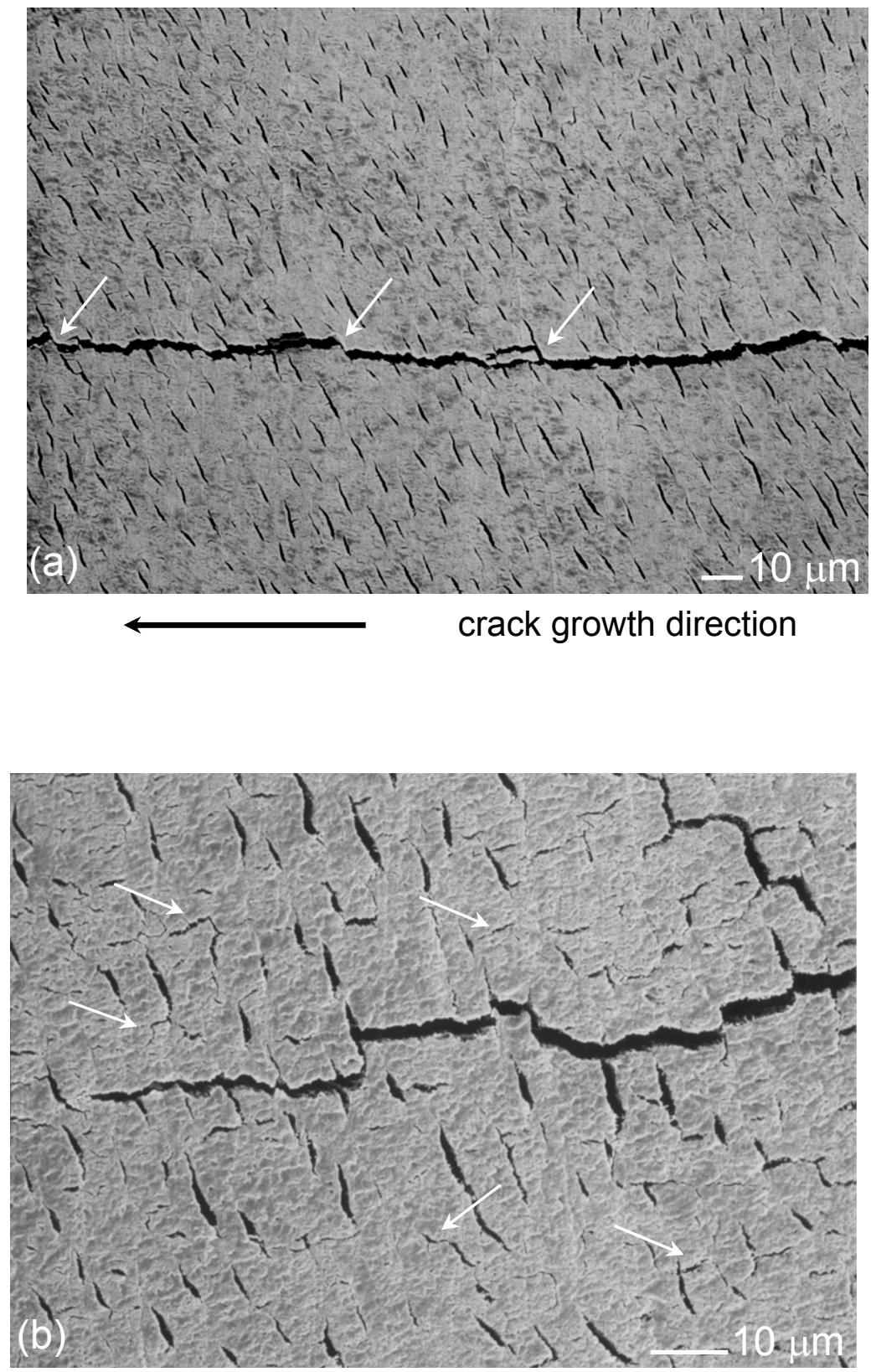

crack growth direction

Fig. 9: Scanning electron micrographs of typical crack paths for the nominally perpendicular orientation in the context of crack- microstructure interactions. The white arrows indicate in (a) the slight out-of-plane deflections made by the crack, and in (b) microdamage in the form of microcracks, a possible toughening mechanism. 

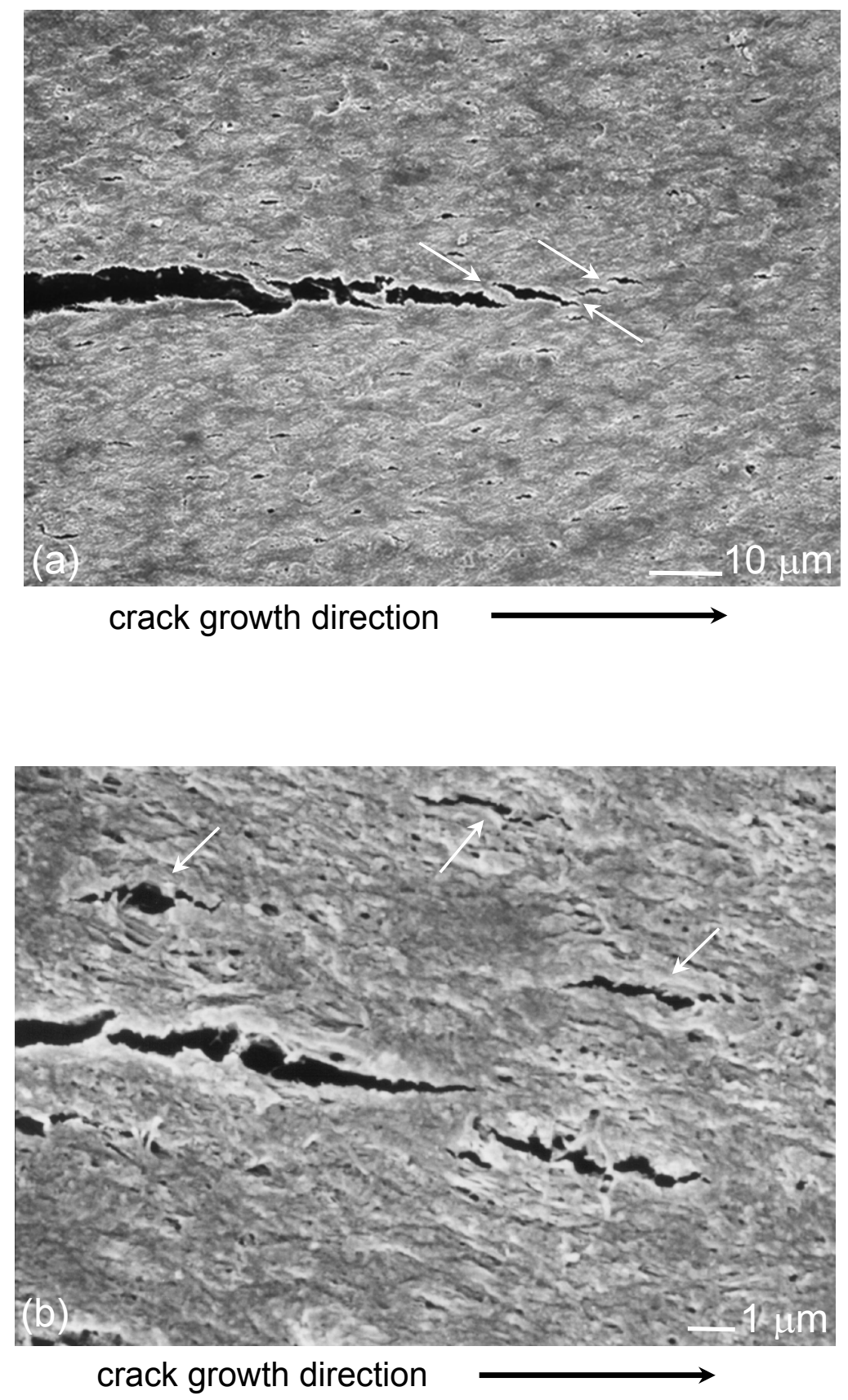

Fig. 10: Scanning electron micrographs of typical crack paths for the nominally anti-plane parallel orientation in the context of crack-microstructure microstructure interactions. The white arrows indicate in (a) uncracked ligament bridging, and in (b) microcracks in the vicinity of the crack. 

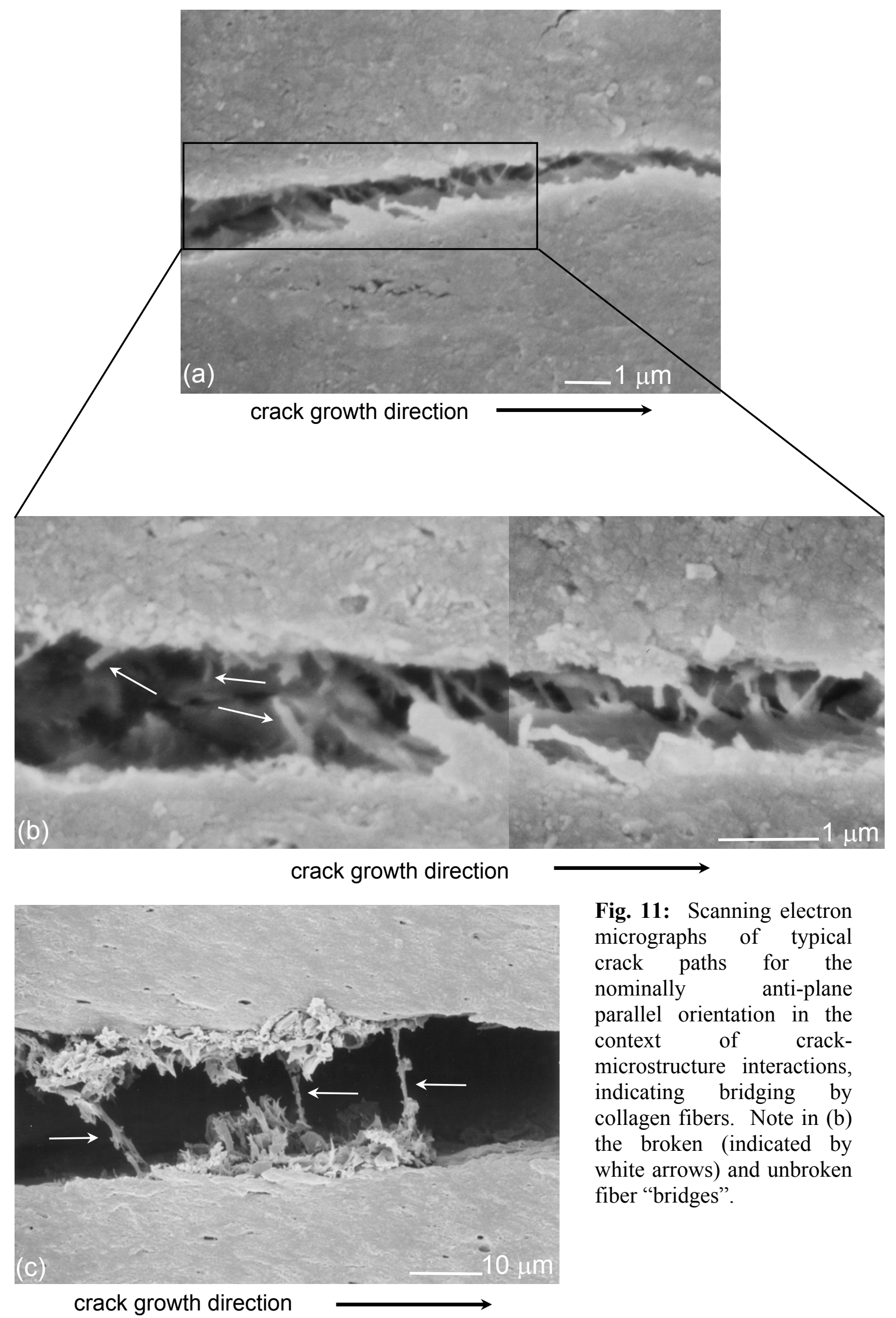

Fig. 11: Scanning electron micrographs of typical crack paths for the nominally anti-plane parallel orientation in the context of crackmicrostructure interactions, indicating bridging by collagen fibers. Note in (b) the broken (indicated by white arrows) and unbroken fiber "bridges". 


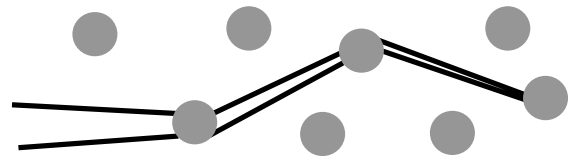

(a) Crack Deflection

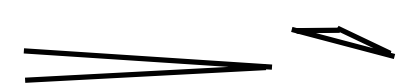

(c) Uncracked Ligament Bridging

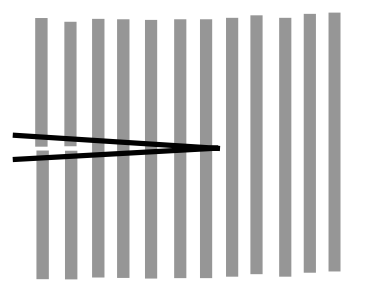

(b) Crack Bridging

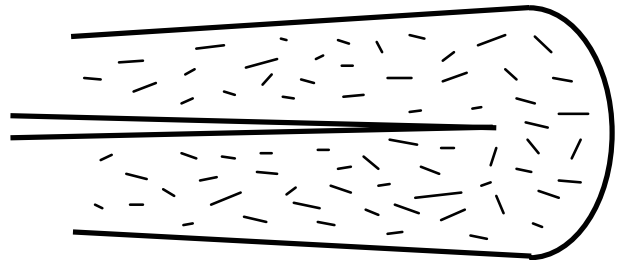

(d) Microcracking

Fig. 12: Schematic illustrations of some of the possible toughening mechanisms possible in dentin: (a) crack deflection, (b) crack bridging (by collagen fibers), (c) uncracked ligament bridging, and (d) microcracking. 